\title{
Analysis and correction to the influence of satellite motion on the measurement of inter-satellite two-way clock offset
}

\author{
Feijiang Huang ${ }^{1,2,4}$, Yanyu Chen ${ }^{1,2}$, Tinghui Li ${ }^{2}$, Qingxiao Shan ${ }^{1}$, Junjun Zhang ${ }^{1}$ and Chenglin $\mathrm{Cai}^{3,4^{*}}$
}

\begin{abstract}
Aiming at the influence of satellite motion on the inter-satellite two-way clock offset measurement, this paper proposed a correction method to eliminate the influence of satellite motion. Firstly, the measuring principle for the inter-satellite two-way clock offset was offered, and then specific influences of satellite motion on the measurement of inter-satellite two-way clock offset were analyzed in details when the mutual closing and faraway motions were conducted among the satellites. Finally, this paper integrated the specific influences of two motions on the measurement of inter-satellite clock offset, and put forward the correction method of inter-satellite clock offset with the smallest error after the polynomial fitting of measured clock offset. Satellite tool kit (STK) was used for the simulation of two-way clock offset measurement, and the result indicated that the accuracy of measurement for the inter-satellite clock offset with the length of 8 min containing the simulation error was better than $1 \mathrm{~ns}$. The clock offset correction method can be used for the autonomous and high-accuracy measurement of inter-satellite clock offset.
\end{abstract}

Keywords: Satellite communication, Satellite constellation, Satellite motion, Time synchronization, Clock offset measurement

\section{Introduction}

Rapid development of space technology have witnessed diversified forms that are displayed for various space applications where one typical representatives are satellite navigation system [1-4], space-based TT\&C [5, 6], manned spaceflight [7], deep space exploration [8], and distributed satellite system and others [9]. These aerospace application systems are dependent on the spacecraft, navigation satellite, explorer satellite, other various special satellites, small satellites in formation flying, and others. To realize timely sharing and comprehensive utilization, we must obtain information among different systems, and the unified time reference must be equipped among the systems. In addition, one precondition to establish the high-precision time reference of the system is to get

\footnotetext{
* Correspondence: csdxgxg|@sina.com

${ }^{3}$ School of Information and Communication, Guilin University of Electronic Technology, Guilin 541004, China

${ }^{4}$ Guangxi Colleges and Universities Key Laboratory of Satellite Navigation and Position Sensing, Guilin 541004, China

Full list of author information is available at the end of the article
}

the high-precision clock offset of each satellite within the system.

Through comparison with ground station, we can realize the measurement of clock offset, and in current aerospace application, the time reference is established on the ground. But such measurement has several shortcomings: short measurement time, low measurement precision, and others limitations resulting from short visual time between the ground station and satellite. Moreover, once the ground station is damaged, that measurement of clock offset cannot be conducted. After establishing inter-satellite link for the measurement of clock offset directly in the inter-satellite, we can obtain high-precision system time reference, get high-precision inter-satellite clock offset, and use the suitable algorithm processing. The satellite-ground two-way time synchronization is one of the methods to measure the high-precision clock offset between the satellite and ground station [10-12]. It can improve the measurement precision of clock offset because it can offset the influence of additional delay at 
atmospheric layer, as well as the time delay between the satellite and ground station. However, the satellite and ground are required to stay in a relative rest state when we use this method; otherwise, the inconsistent two-way time delay caused by the satellite motion will affect the accuracy. This paper used satellite-ground two-way time synchronization method for autonomous measurement of inter-satellite clock offset, and analyzed the specific influence of satellite motion on the measurement. Taking the satellite motion into consideration, this paper deduced the correction method of inter-satellite two-way clock offset measurement, and the correctness of the method was verified via satellite tool kit (STK) simulation.

\section{Methods and experiments}

\subsection{Method of measuring inter-satellite two-way clock offset}

Clock offset measurement with inter-satellite two-way time synchronization is shown in Fig. 1 [12]. The clock offset between satellite $\mathrm{A}$ and $\mathrm{B}$ is set as $\Delta t$. Satellite A and B participating in the measurement of clock offset transmit the timing signal via own sending equipment respectively. The following equation can be obtained after the timing signal from the other party is received via the receiving equipment.

$$
\begin{aligned}
& T_{1}=\Delta t+t_{2}+\tau_{B A}+r_{1}+\delta_{1} \\
& T_{2}=-\Delta t+t_{1}+\tau_{A B}+r_{2}+\delta_{2}
\end{aligned}
$$

In the equation, $T_{1}$ is the time difference between the timing signals transmitted by satellite $\mathrm{A}$ and received from satellite $\mathrm{B} ; t_{1}$ is the time delay of transmitting equipment for satellite $\mathrm{A} ; r_{1}$ is the time delay of receiving equipment for satellite $\mathrm{A} ; \tau_{\mathrm{BA}}$ is the propagation time delay from satellite $\mathrm{B}$ to satellite $\mathrm{A}$; $\delta_{1}$ is other time delay; $T_{2}$ is the time difference between the timing signals transmitted by satellite $B$ and received from satellite $A ; t_{2}$ is the time delay of transmitting equipment for satellite $\mathrm{B} ; r_{2}$ is the time delay of receiving equipment for satellite $\mathrm{B}$; $\tau_{\mathrm{AB}}$ is the propagation time delay from satellite $A$ to satellite $B$; and $\delta_{2}$ is other time delay. The clock offset $\Delta$ tof two satellites can be obtained from Eqs. (1) and (2) as shown below.

$$
\Delta t=\frac{T_{1}-T_{2}}{2}+\frac{t_{1}-t_{2}}{2}+\frac{r_{2}-r_{1}}{2}+\frac{\tau_{A B}-\tau_{B A}}{2}+\frac{\delta_{2}-\delta_{1}}{2}
$$

In Eq. (3), $T_{1}$ can be measured via satellite $A ; T_{2}$ can be measured via satellite $\mathrm{B}$. According to the frequency of transmitting signal of the satellite, $t_{1}, t_{2}, r_{1}$, and $r_{2}$ can be calibrated in advance; therefore, the time delay can be processed as the known value, and the measurement influence on the clock offset can be ignored. In order to highlight the specific influence of satellite motion on measuring inter-satellite clock offset, the satellite clock offset $\Delta t$ is supposed as unchanged in the measurement process of clock offset, and the simplified calculation formula for clock offset can be obtained as the following.

$$
\Delta t=\frac{T_{1}-T_{2}}{2}+\frac{\tau_{A B}-\tau_{B A}}{2}+\frac{\delta_{2}-\delta_{1}}{2}
$$

Seen from formula (4), the precision of inter-satellite clock offset measurement is mainly depending on three main factors of measurement: precision on time difference $\left(T_{1}-T_{2}\right) / 2$, transmission time delay difference $\left(\tau_{A B^{-}} \tau_{B A}\right) / 2$ of timing signal, and other delay difference $\left(\delta_{2}-\delta_{1}\right) / 2$. When the clock offset measurement for satellite $\mathrm{A}$ and $\mathrm{B}$ is conducted at the frequency of higher than once per second, the influence of other time delay $\left(\delta_{2}-\delta_{1}\right) / 2$ on the precision of clock offset measurement is contained into the time difference and time delay difference. Therefore, in order to analyze the influence of satellite motion on the precision of clock offset measurement, we should consider the close frequency of timing signals

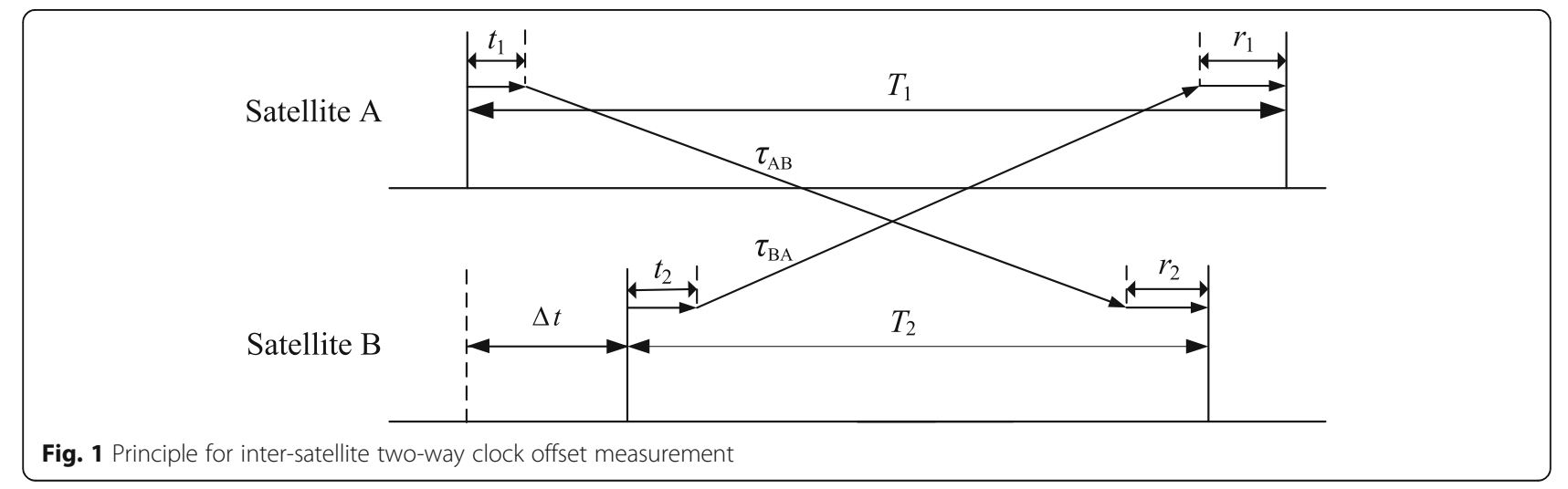


transmitted by each party at the same time and the basic same of the path passed by the timing signal. Formula (4) can be further simplified as the following.

$$
\Delta t=\frac{T_{1}-T_{2}}{2}+\frac{\tau_{A B}-\tau_{B A}}{2}
$$

Meanwhile, formula (1) and (2) can be changed as

$$
\begin{aligned}
& T_{1}=\Delta t+\tau_{B A} \\
& T_{2}=-\Delta t+\tau_{A B}
\end{aligned}
$$

Formula (6) and (7) are the basic formula analyzing the influence of satellite motion on the precision of inter-satellite two-way clock offset. If the location of the satellite for two-way clock offset measurement in the measuring process does not change, the clock offset of two satellites can be obtained from formula (5) as the following.

$$
\Delta t=\frac{T_{1}-T_{2}}{2}
$$

\subsection{The influence of satellite motion on the clock offset measurement and correction method}

According to the simulation of satellite constellation, the main performance of inter-satellite motion within the constellation are the mutual close, mutual far-away, and relatively unchanged location $[13,14]$. Therefore, on the influence of satellite motion on the inter-satellite two-way clock offset measurement, the mutual close and mutual far-away are only considered for the following analysis. In order to simplify the analysis, the initial inter-satellite distance of satellite is $R$; the speed from satellite A to satellite $\mathrm{B}$ is $v_{A}$; and the speed from satellite $\mathrm{B}$ to satellite $\mathrm{A}$ is $v_{B}$. Then, $v_{A}<v_{B}$ supposing the inter-satellite motion is rectilinear motion.

When the mutual close motion is conducted among the satellites, the schematic diagram is as shown in Fig. 2.
According to the principle of invariance of light velocity, it can be obtained from the figure.

$$
\begin{aligned}
& c \cdot \tau_{B A}+v_{A} \cdot \tau_{B A}=R \\
& c \cdot \tau_{A B}+v_{B} \cdot \tau_{A B}=R
\end{aligned}
$$

It can be obtained from formula (9) and (10).

$$
\begin{aligned}
\tau_{B A} & =\frac{R}{c+v_{A}} \\
\tau_{A B} & =\frac{R}{c+v_{B}}
\end{aligned}
$$

Substituting formula (6) with formula (11), and formula (7) with formula (12), we can get the following.

$$
\begin{aligned}
& T_{1}=\Delta t+\frac{R}{c+v_{A}} \\
& T_{2}=-\Delta t+\frac{R}{c+v_{B}}
\end{aligned}
$$

The calculation formula for the clock offset at the mutual close of satellite can be obtained from formula (13) and (14).

$$
\begin{aligned}
\Delta t & =\frac{T_{1}-T_{2}}{2}+\frac{1}{2} \cdot\left(\frac{R}{c+v_{B}}-\frac{R}{c+v_{A}}\right) \\
& =\frac{T_{1}-T_{2}}{2}+\frac{R\left(v_{A}-v_{B}\right)}{2 \cdot\left(c+v_{B}\right) \cdot\left(c+v_{A}\right)}
\end{aligned}
$$

Formula (15) is the influence of satellite motion on inter-satellite clock offset $\Delta t$ at the mutual close of the satellite. Taking the influence of satellite motion on the measurement of inter-satellite two-way clock offset out of consideration, we can see from formula (15), if $v_{A}<v_{B}$, the value of $\frac{R\left(v_{A}-v_{B}\right)}{2 \cdot\left(c+v_{B}\right) \cdot\left(c+v_{A}\right)}$ is negative, which indicates that the calculation result would be bigger than the actual clock offset calculated via formula (8). In order to correct the

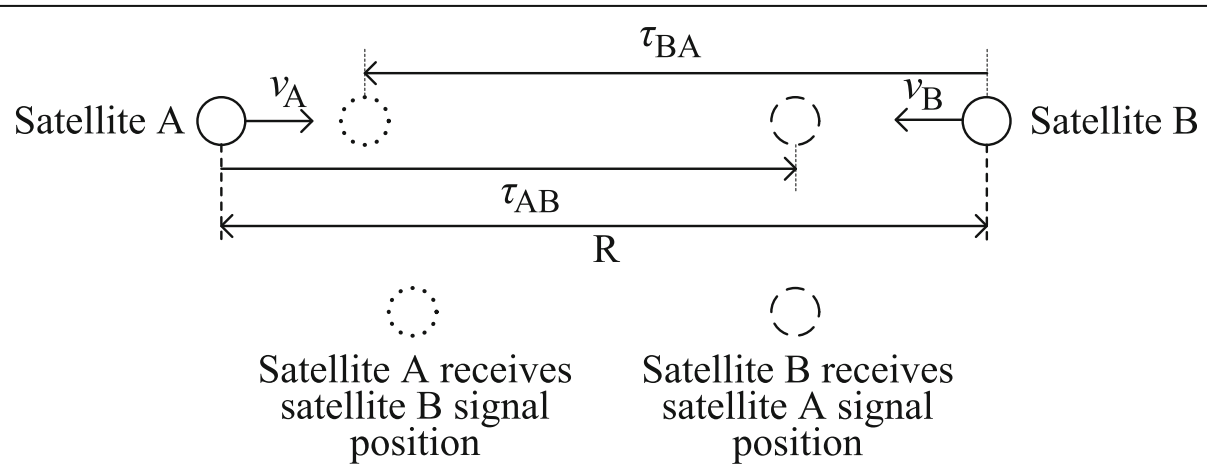

Fig. 2 Influence of inter-satellite mutual close on the measurement of inter-satellite two-way clock offset 
big calculation result, the corrected value is negative $\frac{R\left(v_{A}-v_{B}\right)}{2 \cdot\left(c+v_{B}\right) \cdot\left(c+v_{A}\right)}$.

When the mutual far-away motion is conducted among the satellites, the schematic diagram is as shown in Fig. 3. According to the principle of invariance of light velocity, it can be obtained from the figure.

$$
\begin{aligned}
& c \cdot \tau_{B A}-v_{A} \cdot \tau_{B A}=R \\
& c \cdot \tau_{A B}-v_{B} \cdot \tau_{A B}=R
\end{aligned}
$$

It can be obtained from formula (16) and (17).

$$
\begin{aligned}
& \tau_{B A}=\frac{R}{c-v_{A}} \\
& \tau_{A B}=\frac{R}{c-v_{B}}
\end{aligned}
$$

Substituting formula (6) with formula (18), and formula (7) with formula (19), we can get the following.

$$
\begin{aligned}
& T_{1}=\Delta t+\frac{R}{c-v_{A}} \\
& T_{2}=-\Delta t+\frac{R}{c-v_{B}}
\end{aligned}
$$

The calculation formula for the clock offset at the mutual far-away of satellite can be obtained from formula (20) and (21).

$$
\begin{aligned}
\Delta t & =\frac{T_{1}-T_{2}}{2}+\frac{1}{2} \cdot\left(\frac{R}{c-v_{B}}-\frac{R}{c-v_{A}}\right) \\
& =\frac{T_{1}-T_{2}}{2}+\frac{R\left(v_{B}-v_{A}\right)}{2 \cdot\left(c-v_{B}\right) \cdot\left(c-v_{A}\right)}
\end{aligned}
$$

Formula (22) is the influence of satellite motion on inter-satellite clock offset $\Delta t$ at the mutual far-away of the satellite. Taking the influence of satellite motion on the measurement of inter-satellite two-way clock offset out of consideration, we can see from formula (22), if $v_{A}<v_{B}$, the value of $\frac{R\left(v_{B}-v_{A}\right)}{2 \cdot\left(c-v_{B}\right) \cdot\left(c-v_{A}\right)}$ is positive, which indicates that the calculation result will be smaller than the actual clock offset calculated via formula (8). In order to correct the small calculation result, the corrected value is positive $\frac{R\left(v_{B}-v_{A}\right)}{2 \cdot\left(c-v_{B}\right) \cdot\left(c-v_{A}\right)}$.

\subsection{Correction method of the inter-satellite clock offset measurement}

Seen from formula (15) and (22), the correction value is $\frac{R\left(v_{A}-v_{B}\right)}{2 \cdot\left(c+v_{B}\right) \cdot\left(c+v_{A}\right)}$ at the inter-satellite close motion, and it is negative. The correction value is $\frac{R\left(v_{B}-v_{A}\right)}{2 \cdot\left(c-v_{B}\right) \cdot\left(c-v_{A}\right)}$ at the far-away motion, and it is positive. Therefore, the caused correction value of clock offset is changed from the negative to the positive from the close motion to the far-away motion. When the correction value is 0 , the inter-satellite clock offset from the two-way measurement is nearest to the actual clock offset, and the moment occurred at the turning point from the mutual close to mutual far-away between two satellites. The inter-satellite distance change rate at the moment is 0 via the simulation result.

Via the above error analysis on the measurement result of the satellite motion on inter-satellite two-way clock offset, we can see that, excluding the influence of satellite motion on the clock offset measurement, the clock offset is larger than the actual clock offset at the mutual close motion. The clock offset is smaller than the actual clock offset at the mutual far-away motion if the influence of satellite motion on the clock offset measurement is not considered. If the satellite two-way clock offset measurement is made from the mutual close to mutual far-away, and the influence of satellite motion on the clock offset measurement is not considered, the inter-satellite error clock will change from large to small. The moment for the consistency between measured clock offset and actual clock offset occurs in the process, and the inter-satellite distance change rate among two

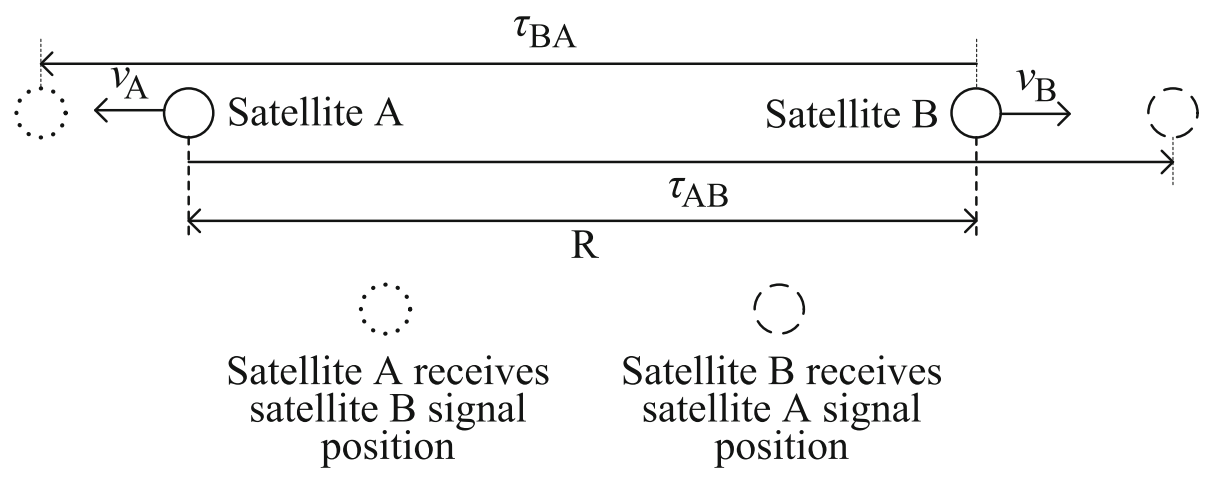

Fig. 3 Influence of inter-satellite mutual far-away motion on the measurement of inter-satellite two-way clock offset 
satellites is 0 at the moment. Therefore, the fitting polynomial of satellite clock offset in the process can be obtained if the least square fitting is conducted for the clock offset data from the measurement in the process.

$$
\Delta t=f(t)
$$

According to the above analysis on the clock offset measurement error, the requirement on the clock offset measurement precision can be met via one fitting for formula (23). In order to verify the correction method after the occurrence of deviation of measured clock offset caused by the above satellite motion, STK simulation tool is used to generate the clock offset measurement data, and the moment with the inter-satellite distance change rate of 0 in the measurement process of the clock offset is obtained via the simulation. The minimum value between the measured clock offset and actual clock offset can be obtained after substituting the moment into formula (23), and the precision of the correction method can be obtained.

\section{Results and discussion}

\subsection{Simulation results}

Supposing the clock offset between satellite A and B is unchanged in $1 \mu \mathrm{s}$ among the measuring process of inter-satellite two-way clock offset and ignoring the equipment time delay and other time delay of satellite receiver and transmitter, we can see the constellations include three layers of satellites of Geosynchronous Earth Orbit (GEO), Inclined Geo Synchronous Orbit (IGSO), and Medium Earth Orbit (MEO) which are selected as the simulation object. GEO satellite and IGSO satellite are served as satellite A; MEO satellite is served as satellite B; STK is used to simulate 4-section inter-satellite clock offset measured data respectively. After the clock offset is calculated with formula (8) along with the satellite motion change rule, one polynomial fitting is conducted for the clock offset data calculated in the process. The moment with the inter-satellite distance change rate of 0 is simulated via STK. The inter-satellite clock offset with minimum error is obtained after substituting the moment into the clock offset fitting polynomial, and the verification result of the correction method is obtained via the comparison between the clock offset and supposed clock offset. The comparison result between the inter-satellite clock offset via specific simulation calculation and supposed actual clock offset is shown in Table 1.

It is indicated in Table 1 that when the time period for inter-satellite two-way clock offset measurement is basically symmetric compared with the moment at the inter-satellite distance change rate of 0 , the fitting precision measured for clock offset fit polynomial is higher with small fitting error and high clock offset measuring precision, and the minimum error between the calibrated inter-satellite clock offset and supposed clock offset including the simulation error condition is 0.287340679374 ns. The precision for measured clock offset fit polynomial is lower with large fitting error and reduced clock offset measuring precision when the time period for inter-satellite two-way clock offset measurement is asymmetric compared with the moment at the inter-satellite distance change rate of 0 . The above results show that the proposed correction method for inter-satellite clock offset is correct.

\subsection{Results discussion}

Same with the satellite-ground two-way time synchronization, we take the different deviation occurs at each simulation experimental result during the simulation period of inter-satellite two-way clock offset measurement for the influence of satellite motion direction and Sagnac effect on the correction method out of consideration [14]. The satellite motion directions at the same time length and different time period as well as different time length and different time period are different when simulating and calculating the clock offset data, and the influence on the clock offset fit polynomial error is also different. Especially, the deviation between the clock offset after the correction and actual clock offset is the maximum when the time period for clock offset measurement is asymmetric compared with the moment at the inter-satellite distance change rate of 0 . At the actual application of the correction method, the simulation influence on the correction method is reduced for the influence of satellite motion direction and Sagnac effect on the algorithm is contained in the measuring value of $T_{1}$ and $T_{2}$ [15]. If the high-precision time difference measuring method is used for the moving satellite and the data processing method with higher fitting precision is used, the precision of correction method can be improved further, and the actual clock offset measurement precision will be higher than the result obtained from the simulation.

\section{Conclusions}

In this paper, the specific error formula for the inter-satellite motion on the two-way clock offset measurement is deduced in details based on the measuring principle of inter-satellite two-way clock offset. The specific influence of satellite motion on the inter-satellite two-way clock offset measurement is analyzed with the formula, and the calibration method eliminating the influence of satellite motion is proposed. The simulation result of inter-satellite clock offset measurement indicates that the least square one fitting is used in the calibration method to process the inter-satellite clock offset 


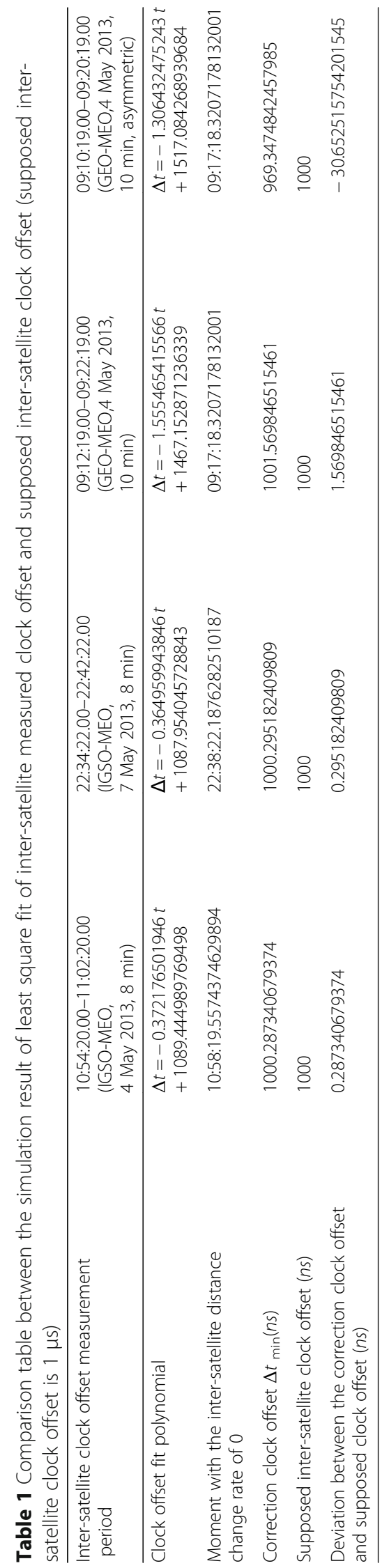


data. The precision of clock offset measurement is better than $1 \mathrm{~ns}$ when the time period for clock offset measurement is basically symmetric compared with the moment at the inter-satellite distance change rate of 0 and the influence of simulation error is contained. If the data processing method with higher precision is used in the actual inter-satellite clock offset measurement, the precision of clock offset measurement can be further improved to meet the high-precision demand on inter-satellite independent clock offset measurement for various moving satellites. The satellite clock offset prediction algorithm with higher precision will be used in the future to further improve the correction ability of the algorithm on the influence of satellite motion combined with the inter-satellite clock offset measured data processing algorithm.

\section{Abbreviations}

GEO: Geosynchronous Earth Orbit; IGSO: Inclined geosynchronous orbit: MEO: Medium Earth Orbit; STK: Satellite tool kit

\section{Acknowledgements}

The authors would like to thank all kinds of funds for funding.

\section{Funding}

This study was supported by the National Natural Science Foundation of China (Nos.11373075, 61771150, 61264008), Hunan Provincial Natural Science Foundation of China (No.2015JJ2016), Guangxi Colleges and Universities Key Laboratory of Satellite Navigation and Position Sensing, Youth Talent Support Plan and Scientific Research Foundation of Changsha University (No.SF1615).

\section{Availability of data and materials}

Data sharing not applicable to this article as no datasets were generated or analyzed during the current study.

\section{Authors 'contributions}

$\mathrm{FH}$ and CC carried out the conception studies and algorithm design, participated in drafted the manuscript. YC and JZ carried out the simulation experiment. TL and QS carried out the error analysis of the experiment results. All authors read and approved the final manuscript.

\section{Competing interests}

The authors declare that they have no competing interests.

\section{Publisher's Note}

Springer Nature remains neutral with regard to jurisdictional claims in published maps and institutional affiliations.

\section{Author details}

${ }^{1}$ College of Electronic Information and Electrical Engineering, Changsha University, Changsha 410022, China. ${ }^{2}$ College of Electronic Engineering, Guangxi Normal University, Guilin 541004, China. ${ }^{3}$ School of Information and Communication, Guilin University of Electronic Technology, Guilin 541004, China. ${ }^{4}$ Guangxi Colleges and Universities Key Laboratory of Satellite Navigation and Position Sensing, Guilin 541004, China.

Received: 17 July 2018 Accepted: 21 December 2018

Published online: 07 January 2019

\section{References}

1. Y. Yang, Y. Xu, J. Li, C. Yang, Progress and performance evaluation of BeiDou global navigation satellite system: data analysis based on BDS-3 demonstration system. Sci. China. Earth. Sci. 61(5), 614-624 (2018)

2. Z. Yanan, L. Ruihua, W. Jian, Research on time domain evaluation method of BDS B1 band signal quality. Chinese. Space. Sci. Technol. 38(01), 29-35 (2018)
3. Z. Xingyu, C. Hua, A. Xiangdong, Analysis of Galileo signal quality and positioning performance. Gnss. World. China. 43(01), 19-24 (2018)

4. Z. Anmin, Y. Yang, J. Yifan, M. Feng, Systematic Bias compensation model of inter-system bias and its performance analysis for BDS/GPS fusion positioning. Geomatics. Inf. Sci. Wuhan. Univ. 42(10), 1423-1430 (2017)

5. Y. Hongjun, Latest development progress and trends of foreign data relay satellite systems. Telecommun. Eng. 56(01), 109-116 (2016)

6. L. Li, Development status and trends of space TT\&C and communication technology. Telecommun. Eng. 57(12), 1464-1470 (2017)

7. L. Xiaogang, W. Yansong, S. Yao, Review of human spaceflight development abroad in 2017. Manned. Spaceflight. 24(02), 279-284 (2018)

8. S. Zezhou, M. Linzhi, Current situation and sustainable development trend of deep space exploration in China. J. Nanjing. Univ. Aeronaut. Astronaut. 47(06), 785-791 (2015)

9. L. Yong, Observability of autonomous navigation of distributed satellite system using relative position measurements. Scientia. Sinica(Mathematica). 46(10), 1461-1472 (2016)

10. T. Guifen, W. Yang, S. Ranran, X. Aimin, Time synchronization method base on combined satellite-ground and inter-satellite observation. Geomatics. Inf. Sci. Wuhan. Univ. 43(02), 183-187 (2018)

11. L. Hongchun, L. Xiaochun, W. Jianfeng, A method of two-way satelliteground time synchronization under inter-satellite links system. J. Astron. 38(07), 728-734 (2017)

12. H. Feijiang, L. Xiaochun, L. Guangcan, S. Liping, Z. Wenxi, Y. Cheng, An algorithm for dynamic satellite-ground two-way time synchronization and ranging. J. Astronaut. 35(09), 1050-1057 (2014)

13. H. Feijiang, J. Yang, X. Lu, S. Qingxiao, Z. Yongbin, C. Jianyun, H. Zhuli, An inter-satellite dynamic ranging algorithm based on two-way time synchronization. Comput. Model. New. Technol. 18(10), 19-24 (2014)

14. $\mathrm{H}$. Bo, H. Xiulin, Inter-satellite ranging and time synchronization technique for BD2. J. Astronaut. 32(06), 1271-1275 (2011)

15. L. Yong, Sagnac effect on two-way time synchronization between satellites. Space. Electron. Technol. 2, 46-49 (2013)

\section{Submit your manuscript to a SpringerOpen ${ }^{\circ}$ journal and benefit from:}

- Convenient online submission

- Rigorous peer review

- Open access: articles freely available online

High visibility within the field

- Retaining the copyright to your article

Submit your next manuscript at $>$ springeropen.com 